\title{
A functional relationship between helix 1 and the 900 tetraloop of $16 S$ ribosomal RNA within the bacterial ribosome
}

\author{
FRANÇOIS BÉLANGER, ${ }^{1}$ GABRIEL THÉBERGE-JULIEN, ${ }^{1}$ PHILIP R. CUNNINGHAM, ${ }^{2}$ \\ and LÉA BRAKIER-GINGRAS ${ }^{1}$ \\ ${ }^{1}$ Département de Biochimie, Université de Montréal, Montréal, Québec, Canada H3T 1J4 \\ ${ }^{2}$ Department of Biological Sciences, Wayne State University, Detroit, Michigan 48202, USA
}

\begin{abstract}
The conserved 900 tetraloop that caps helix 27 of 165 ribosomal RNA (rRNA) interacts with helix 24 of 165 rRNA and also with helix 67 of 235 rRNA, forming the intersubunit bridge B2c, proximal to the decoding center. In previous studies, we investigated how the interaction between the 900 tetraloop and helix 24 participates in subunit association and translational fidelity. In the present study, we investigated whether the 900 tetraloop is involved in other undetected interactions with different regions of the Escherichia coli 16S rRNA. Using a genetic complementation approach, we selected mutations in 16S rRNA that compensate for a 900 tetraloop mutation, A900G, which severely impairs subunit association and translational fidelity. Mutations were randomly introduced in $16 \mathrm{~S}$ rRNA, using either a mutagenic XL1-Red $E$. coli strain or an error-prone PCR strategy. Gain-offunction mutations were selected in vivo with a specialized ribosome system. Two mutations, the deletion of U12 and the U12C substitution, were thus independently selected in helix 1 of 16S rRNA. This helix is located in the vicinity of helix 27 , but does not directly contact the 900 tetraloop in the crystal structures of the ribosome. Both mutations correct the subunit association and translational fidelity defects caused by the A900G mutation, revealing an unanticipated functional interaction between these two regions of $16 S$ rRNA.
\end{abstract}

Keywords: 16S rRNA 900 tetraloop; $16 S$ rRNA helix 1; 165 rRNA helix 27; central pseudoknot; subunit association; translational fidelity

\section{INTRODUCTION}

The ribosome is a complex ribonucleoprotein responsible for protein synthesis in all organisms. The elucidation of crystal structures of the ribosome and of its subunits has given unprecedented insights into the intricate folding of ribosomal RNA (rRNA) and its interaction with ribosomal proteins (Ban et al. 2000; Schluenzen et al. 2000; Wimberly et al. 2000; Harms et al. 2001; Yusupov et al. 2001). However, high-resolution X-ray structures provide only static pictures of the ribosome, which undergoes important conformational changes during the different steps of protein synthesis. Cryoelectron microscopy (cryo-EM) has proven very useful to study the structure of the ribosome in different functional

Reprint requests to: Léa Brakier-Gingras, Département de Biochimie, Université de Montréal, 2900, boul. Édouard-Montpetit, D-353, Montréal, Québec, Canada H3T 1J4; e-mail: lea.brakier.gingras@umontreal.ca; fax: (514) 343-2210.

Article published online ahead of print. Article and publication date are at http://www.rnajournal.org/cgi/doi/10.1261/rna.2160405. states (Frank and Agrawal 2000; van Heel 2000), but its resolution is limited to $7 \AA$. Detailed structures of all the functional states of the ribosome will be required to understand its dynamics and the interplay between the different rRNA regions as well as between rRNA and proteins.

The study of the crystal structure of the 30S subunit from Thermus thermophilus, complexed with mRNA and cognate tRNA fragments or with paromomycin, an error-inducing antibiotic, revealed how the decoding center of the $30 \mathrm{~S}$ subunit recognizes cognate tRNAs using an induced-fit mechanism (Ogle et al. 2001, 2002, 2003). Upon binding of a tRNA to the ribosome, nucleotides A1492 and A1493 flip out of helix 44, while nucleotide G530 of helix 18 switches from a syn to an anti conformation, so as to interact with the minor groove of the codon-anticodon duplex. Recognition of a cognate codon-anticodon interaction triggers a conformational rearrangement of the whole $30 \mathrm{~S}$ subunit, which changes from an open conformation to a more compact one, the closed conformation, with the head and shoulder closing around the A-site tRNA (Ogle et al. 2001, 2002, 2003). In an independent crystallographic study of the Escherichia coli 70 S ribosome, the 
movement of the head was related to subunit association (Vila-Sanjurjo et al. 2003).

Another important player in tRNA selection is helix 27 of $16 \mathrm{~S}$ rRNA. Helix 27 is capped by a highly conserved GCAA tetraloop, the 900 tetraloop, which docks into the minor groove of the 769-810 region in helix 24 of 16S rRNA (Schluenzen et al. 2000; Wimberly et al. 2000). The other end of helix 27 contacts helix 44 close to the decoding center (Schluenzen et al. 2000; Wimberly et al. 2000). Helix 27 was previously proposed to undergo a base-pairing rearrangement during tRNA selection (Lodmell and Dahlberg 1997). Further studies did not support that this rearrangement is associated with tRNA selection (Velichutina et al. 2000; Vila-Sanjurjo et al. 2003; Rodriguez-Correa and Dahlberg 2004), but did not exclude the possibility that it could occur during translation. Nevertheless, a role for helix 27 and its capping loop in translational accuracy is supported by mutagenesis studies (Velichutina et al. 2000; Bélanger et al. 2002). The 900 tetraloop is also involved in the formation of the intersubunit bridge B2c (Yusupov et al. 2001).

We previously showed that mutations in the 900 tetraloop or mutations in its receptor in helix 24 decrease ribosome activity, impair translational accuracy, and interfere with subunit association. The simultaneous presence of these mutations restore ribosome activity, translational accuracy, and subunit association, indicating that these mutations are complementary (Bélanger et al. 2004). This work demonstrated the functional importance of the interaction between helix 24 and the 900 tetraloop that is detected in the crystal structure of the 30 S subunit. Since the structure of the ribosome is dynamic, it can be suggested that the 900 tetraloop could be involved in alternative interactions during protein synthesis in addition to its interaction with helix 24 . In order to identify direct or indirect interactions not detected in the crystal structures, we carried out genetic complementation experiments, where we selected random mutations in the $16 \mathrm{~S}$ rRNA that complement a deleterious mutation in the 900 tetraloop. We used the weakly active A900G mutant of the 900 tetraloop and introduced random mutations in $16 \mathrm{~S}$ rRNA, using either an XL1-Red mutator strain or an error-prone PCR technique. Gain-of-function mutations were selected in vivo, using a specialized ribosome system (Lee et al. 1997; Morosyuk et al. 2000, 2001). Mutations that compensate for the A900G mutation were thus selected in helix 1 of $16 \mathrm{~S}$ rRNA.

\section{RESULTS}

\section{Selection of mutations in 165 rRNA that complement a mutation in the 900 tetraloop}

In the present study, we first selected random mutations in the 16S rRNA of E. coli that can compensate for the deleterious effect of the A900G mutation in the 900 tetraloop capping helix 27. This selection was made in vivo with the specialized plasmid pASS2-GFP2, which contains a copy of the $16 \mathrm{~S}$ rRNA gene under control of an inducible lac promoter. It also contains two reporter genes, one coding for chloramphenicol acetyltransferase (CAT) and the other for the green fluorescent protein (GFP). The Shine-Dalgarno sequence of the reporter genes and the anti-Shine-Dalgarno sequence of the plasmidencoded 16S rRNA are mutated, so that only the ribosomes containing the plasmid-encoded $16 \mathrm{~S}$ rRNA are able to bind to and translate the CAT and GFP messengers. The CAT reporter gene confers resistance to chloramphenicol and is used to select functional mutants that grow on chloramphenicol. The GFP reporter gene allows for an accurate quantification of the activity of the mutants. For the genetic complementation assays, we used pASS2-GFP2/A900G, a pASS2-GFP2 derivative containing the A900G mutation in the 16S rRNA gene. Random mutations were introduced in the 16S rRNA gene with two independent approaches. The first approach used an XL1-Red mutator strain from Stratagene, which has a rate of mutation 5000 times higher than a wild-type E. coli strain. Plasmid pASS2-GFP2/A900G was propagated in the XL1-Red strain, a pool of randomly mutated plasmids was obtained (see Materials and Methods for details) and was introduced into wild-type E. coli DH5 cells. The second approach used to generate a pool of random mutants was an error-prone PCR method. The 16S rRNA gene from plasmid pASS2-GFP2/A900G was amplified by PCR with the Taq DNA polymerase in the presence of $0.2 \mathrm{mM} \mathrm{MnCl} 2$. Under these conditions, one or two random mutations were introduced per PCR product (data not shown). The PCR products were then used to replace the corresponding $16 \mathrm{~S}$ rRNA sequence in pASS2-GFP2/A900G. Ligation reactions were electroporated into E. coli DH5 cells, and complementary mutations were selected in E. coli DH5 cells from the pools of random mutations obtained with both mutagenesis approaches. The cells were incubated with isopropyl- $\beta$-D-thiogalactopyranoside (IPTG) to induce expression of the 16S rRNA gene. Cells were plated in the presence of $175 \mu \mathrm{g} / \mathrm{mL}$ of chloramphenicol, and surviving clones were picked (the minimal inhibitory concentration of chloramphenicol for the A900G mutant is $100 \mu \mathrm{g} / \mathrm{mL}$ ). The $16 \mathrm{~S}$ rRNA sequence from surviving clones was subcloned in the original pASS2-GFP2/A900G plasmid, so as to eliminate any possible mutation outside of the 16S rRNA sequence. Clones that conserved the phenotype of complementation were sequenced.

With the pool obtained with the XL1-Red strain, about 2 million clones were screened, and 15 surviving clones were sequenced. Nine clones were true revertants, for which A900G was reverted to wild type, and six clones contained a $\mathrm{U} 12 \mathrm{C}$ substitution in addition to the A900G mutation. With the pool obtained with the PCR approach, a total of $\sim 50,000$ transformants were screened, and 17 clones were sequenced. Ten of these clones were true revertants, but in seven clones, in addition to the $A 900 \mathrm{G}$ mutation, a $U$ at position 12 was deleted. Both complementary mutations obtained are located in helix 1. As shown in Figure 1, helix 1 contains three Watson-Crick base pairs, followed by an irregular part 


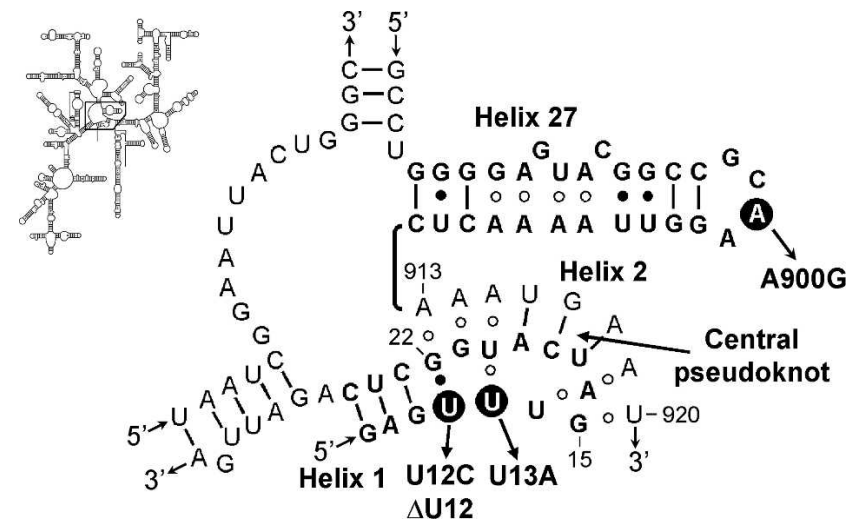

FIGURE 1. Central portion of the secondary structure of E. coli $16 \mathrm{~S}$ rRNA, showing helix 1 and helix 27 in bold. The strand $3^{\prime}$ to helix 27 base pairs with the loop of helix 1 , forming helix 2 , and the central pseudoknot is made of helices 1 and 2 . The secondary structure shown here is derived from the crystal structure of the 30S subunit of $T$. thermophilus. The A900G mutation, the U12C and $\Delta \mathrm{U} 12$ complementary mutations that were selected in this study, and the U13A mutation are indicated.

containing a stretch of three adjacent Us corresponding to nucleotides 12-14. The deletion mutation was designated $\Delta \mathrm{U} 12$, although any of the three Us could be considered as deleted. No other mutation was found, suggesting that helix 1 may be the only region of the 16S rRNA where a single mutation can complement the A900G mutation. Complementary mutations in helix 24 that were previously obtained by site-directed mutagenesis (Bélanger et al. 2004) were not selected in the present study. These are double mutations, and the probability that they occur among the pool of random mutations is very low. It is important to point out that the irregular portion of helix 1 is a part of an important structure called the central pseudoknot of 16S rRNA. As illustrated in Figure 1, this pseudoknot is made of helices 1 and 2, helix 2 being formed by the interaction between residues 913-920, adjacent to helix 27, and the loop of helix 1 . The existence of helix 2 had been first predicted by phylogenetic analysis, which revealed Watson-Crick base pairs between residues 17-19 and 916-918 (Gutell et al. 1994). The crystal structure confirmed these base pairs and showed that helix 2 is longer than initially proposed, with residues 913-915 and 919-920 making non-Watson-Crick base pairs with residues $20-22$ and 15-16, respectively. The importance of the central pseudoknot will be addressed in the Discussion. The stretch of three Us at positions 12-14, where mutations were selected, is highly conserved. The identity of each of these positions is conserved by $>98 \%$ (Comparative RNA Web Site: http://www.rna.icmb.utexas.edu).

\section{Functional studies of mutations in helix 1 of the $16 S$ rRNA}

The effect of the U12C and $\Delta \mathrm{U} 12$ mutations of $16 \mathrm{~S}$ rRNA helix 1 on ribosome activity was examined both in the presence and in the absence of the A900G mutation. Ribosomal activities were measured with the GFP reporter gene of plasmid pASS2-GFP2 (Table 1). As reported previously, the A900G mutation severely decreases the ribosomal activity relative to wild type (7\%). In the presence of the complementary U12C or $\Delta \mathrm{U} 12$ mutation, the GFP activity is increased to $47 \%$ and $37 \%$ relative to wild type, respectively. When these mutations of helix 1 are present alone, they only mildly affect ribosomal activity. The U12C mutant is almost as active as the wild type (90\%), while the $\Delta \mathrm{U} 12$ deletion lowers the ribosomal activity to $70 \%$ of the wild type. Our group previously characterized the U13A mutation at a neighboring position, using a plasmid that contains an $r r n \mathrm{~B}$ operon under the control of a thermoinducible promoter. It was found that this mutation confers resistance to streptomycin, an error-inducing antibiotic, and increases translational fidelity (Pinard et al. 1993, 1994). We decided to investigate whether the U13A mutation also compensates for the A900G mutation. We introduced this mutation in plasmid pASS2-GFP2 using a standard PCR approach and examined its effect on ribosomal activity in the presence and absence of the A900G mutation. As shown in Table 1, the U13A mutation alone has a small effect on ribosomal activity (69\%). When it is combined with the A900G mutation, the resulting activity is $22 \%$, showing that the U13A mutation can also compensate for the A900G mutation, albeit not as efficiently as $\mathrm{U} 12 \mathrm{C}$ or $\Delta \mathrm{U} 12$.

Mutations in the 900 tetraloop decrease translational fidelity and impair subunit association. We investigated the effect of mutations in helix 1 on these two ribosomal functions in the presence or absence of the A900G mutation. The effect of the mutations on subunit association was assessed as previously reported (Bélanger et al. 2002, 2004). The populations of free 30 S subunits, 70 S ribosomes, and polysomes were fractionated from cells expressing the various rRNA mutants. RNA was extracted from each fraction and the proportion of plasmid-encoded $16 \mathrm{~S}$ rRNA was measured using a primer-extension inhibition technique (Sigmund et al. 1988). The results are presented in Figure 2. With a wild-type $16 \mathrm{~S}$ rRNA sequence, the

TABLE 1. Effect of mutations in helix 1 of $16 \mathrm{~S}$ rRNA on ribosome activity in the presence and absence of the A900G mutation

\begin{tabular}{lcc}
\hline & \multicolumn{2}{c}{ GFP activity (\%) } \\
\cline { 2 - 3 } Mutation in helix 1 & A900G & A900 (wild type) \\
\hline None & $7 \pm 1$ & 100 \\
U12C & $47 \pm 2$ & $90 \pm 4$ \\
UU12 & $37 \pm 2$ & $70 \pm 3$ \\
U13A & $22 \pm 1$ & $69 \pm 5$ \\
\hline
\end{tabular}

Ribosomal activities were assessed using a GFP reporter gene and are expressed as a percentage of wild-type activity. Values are the means \pm standard error of three to five independent experiments. 
proportion of plasmid-encoded $16 \mathrm{~S}$ rRNA is about $50 \%$ in each fraction. With the A900G mutation, the proportion of plasmid-encoded $16 \mathrm{~S}$ rRNA in $70 \mathrm{~S}$ ribosomes and polysomes drops to about $10 \%$; we previously demonstrated that this decrease is caused by a defect in subunit association (Bélanger et al. 2004). When the A900G mutation is combined with a complementary mutation in helix 1 , the proportion of plasmid-encoded 16S rRNA in the 70S and polysome fractions increases to levels close to that in the free $30 \mathrm{~S}$ fraction. When mutations in helix 1 are present in the context of a wild-type 900 tetraloop, the plasmidencoded $16 \mathrm{~S}$ rRNA distribution is equivalent in each fraction, as is the case for the wild type. From these observations, we conclude that the mutations in helix 1 correct the subunit association defect caused by the A900G mutation, but have no effect on subunit association on their own.

To study the effect of mutations in helix 1 on translational fidelity, we used derivatives of plasmid pRNAluc2 (Bélanger et al. 2004), a specialized plasmid similar to pASS2-GFP2, except that it contains a copy of the whole $r r n \mathrm{~B}$ operon and not only the 16S rRNA gene. It also harbors a very sensitive reporter gene coding for the firefly luciferase. Plasmid pRNAluc2/UGA and pRNAluc2/-1 contain cassettes in the beginning of the coding sequence of the luciferase gene that introduce a UGA stop codon and a -1 frameshift mutation, respectively. With these plasmids, the production of luciferase requires either a readthrough or -1 frameshift. A third plasmid, pRNAluc2/R218G, contains a R218G mutation in the active center of the luciferase, so that synthesis of active luciferase requires an amino acid misincorporation at this codon. The U12C, $\Delta \mathrm{U} 12$, and U13A mutations, with or without the A900G mutation, were introduced in the sequence of the 16S rRNA gene of these derivatives, and values for the luciferase activities were measured. The results in Figure 3 show that the A900G mutation causes a large increase in the level of

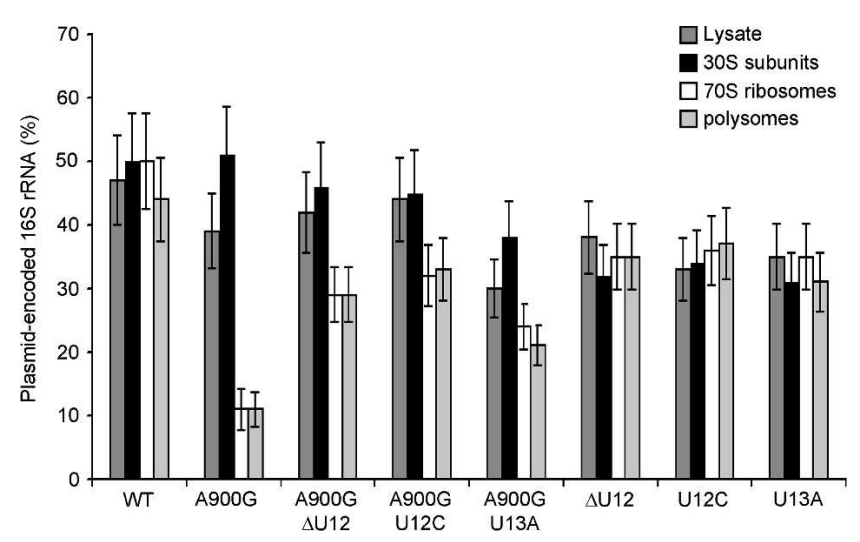

FIGURE 2. Effect of mutations in helix 1 and in the 900 tetraloop on subunit association. The proportion of plasmid-encoded 16S rRNA in the bacterial lysate and in the free $30 \mathrm{~S}$ subunit, $70 \mathrm{~S}$ ribosome, and polysome fractions was assessed by a standard primer extension assay (see Materials and Methods). Results are the means \pm standard error of three independent experiments.

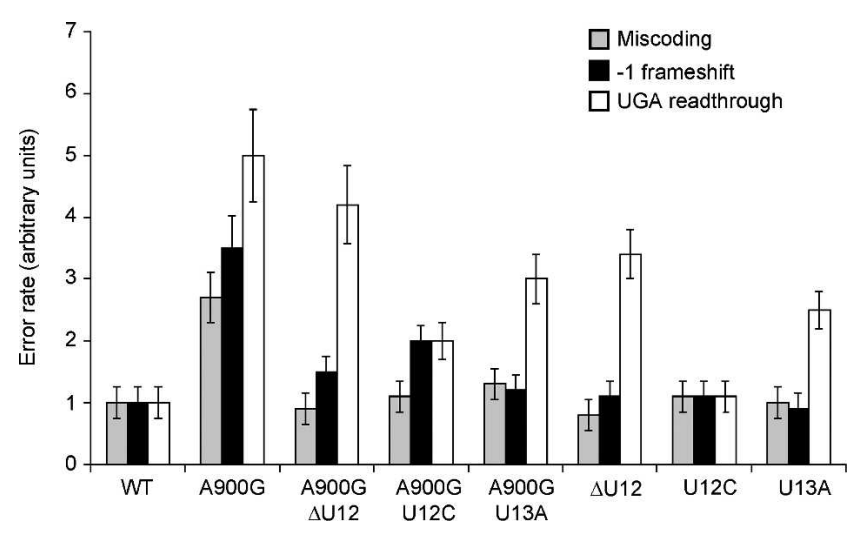

FIGURE 3. Effect of mutations in helix 1 and in the 900 tetraloop on translational fidelity. For each 16S rRNA mutation investigated, the levels of misincorporation, -1 frameshift, and UGA readthrough were determined by dividing the luciferase activity with the derivatives of plasmid pRNAluc2/R218G, pRNAluc2/-1, or pRNAluc2/UGA by that obtained with a wild-type luciferase construct. A value of 1.0 was arbitrarily ascribed to the levels measured with wild-type 16S rRNA (experimental values in percent were, respectively, $0.31( \pm 0.05) \times 10^{-3}$, $0.13( \pm 0.02)$, and $1.0( \pm 0.1)$ for wild-type misincorporation, frameshift, and readthrough). Results are the means \pm standard error of three to five independent experiments.

misincorporation, frameshift, and readthrough. The presence of the complementary mutations in helix 1 restores translational fidelity close to the wild-type level in the miscoding and frameshifting assays. However, for readthrough assays, only the U12C mutation significantly decreases the readthrough caused by the A900G mutation, whereas the $\Delta \mathrm{U} 12$ and U13A mutations do not, or only slightly decrease the readthrough levels. When mutations in helix 1 are examined in the context of a wild-type 900 tetraloop, they do not affect miscoding or frameshift levels, but the $\Delta \mathrm{U} 12$ and U13A mutations increase the readthrough levels. An increased level of readthrough can result from either a decrease in translational fidelity or a defect in the termination step (Murgola et al. 1995). Since the $\Delta \mathrm{U} 12$ and U13A mutations do not change the miscoding and frameshift levels, it is likely that their effect on readthrough is independent of the decoding function. We conclude that mutations in helix 1 do not alter translational fidelity, although they can correct the fidelity defect caused by the A900G mutation. The lack of effect of the U13A mutation on translational fidelity is in disagreement with the study by Pinard et al. (1994), who reported that this mutation increases translational fidelity. This discrepancy will be addressed in the Discussion.

\section{DISCUSSION}

In the present study, we showed that mutations in helix 1 of $16 \mathrm{~S}$ rRNA can compensate for the deleterious effect of the A900G mutation in the 900 tetraloop capping helix 27, implying a functional relationship between these two regions. To get further insight into the relationship between helix 1 
and the 900 tetraloop, we examined these regions in the crystal structure of the $30 \mathrm{~S}$ subunit from $T$. thermophilus (Wimberly et al. 2000). Figure 4 presents the structure of helices 1 and 27 and also that of helix 24, which contains the receptor for the 900 tetraloop. Helix 1 is located in the center of the 30 S subunit, at the junction of the head, body, and platform; helix 24 is on the platform of the 30 S subunit at the subunit interface; and helix 27 is positioned perpendicularly to and between helix 1 and helix 24. The 900 tetraloop interacts with the 768-770/809-811 base pairs of helix 24 . The backbone of nucleotides $900-901$ and that of 770-771 contact helix 67 of 23S rRNA, forming the intersubunit bridge B2c (Yusupov et al. 2001). We showed previously that the A900G mutation causes a steric clash between G900 and G769, which impairs the docking of the 900 tetraloop into its receptor and interferes with the formation of intersubunit bridge B2c (Bélanger et al. 2004). At the other extremity of helix 27, the adjacent segment encompassing nucleotides 913-920 interacts with the loop of helix 1, forming helix 2, as described above, which creates the central pseudoknot of the 30S subunit. Mutations that disrupt the central pseudoknot destabilize the $30 \mathrm{~S}$ subunit and impair subunit association, demonstrating the importance of the central pseudoknot for ribosome function (Brink et al. 1993; Poot et al. 1996). It had been suggested that the central pseudoknot is subject to conformational rearrangements involving alternate base pairings during protein synthesis (Kössel et al. 1990; Leclerc and BrakierGingras 1991), but these rearrangements were not supported by mutagenesis experiments (Pinard et al. 1993; Poot et al. 1998). It can also be observed that helix 1 is packed between ribosomal proteins S5 and S12. Protein S5 is located on the
A

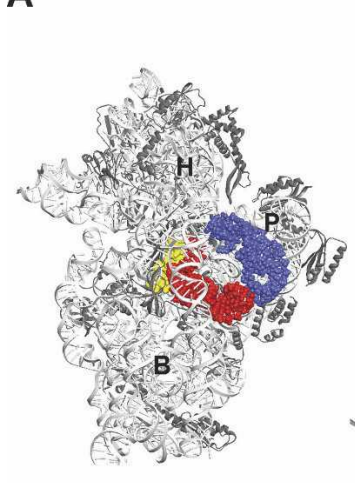

B

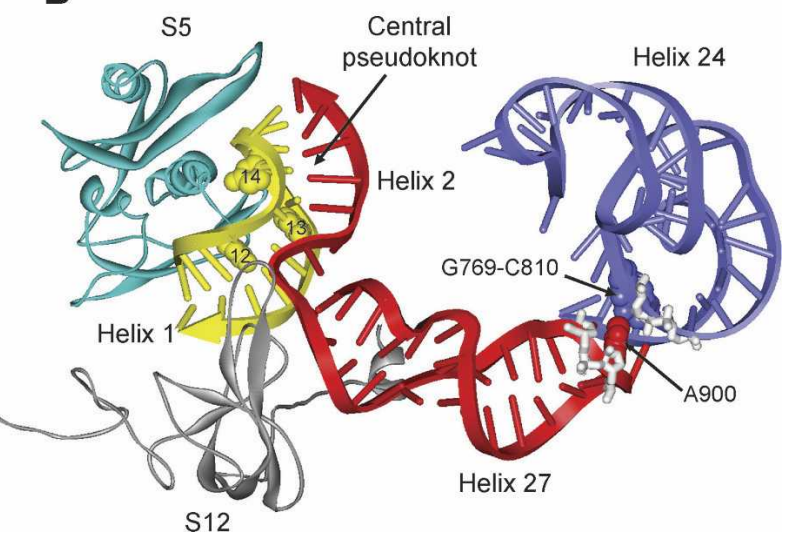

FIGURE 4. Structure of helix 27 and neighboring regions in the crystal structure of the T. thermophilus 30 S subunit (Wimberly et al. 2000, PDB accession number 1FJF). (A) Structure of the $30 \mathrm{~S}$ subunit viewed from the interface. The letters $\mathrm{H}, \mathrm{P}$, and $\mathrm{B}$ indicate, respectively, the head, platform, and body of the subunit. RNA is shown in light gray and proteins in dark gray. Helix 1 is colored in yellow, helix 24 in purple, and helix 27 in red. (B) Details of the structure of helices 24 and 27 and of the central pseudoknot made by helices 1 and 2. Helices are colored as in A. Nucleotides U12-U14, nucleotide A900, and the G769-C810 base pair of helix 24 are shown in space-filling models (yellow, red, and purple, respectively). Ribosomal proteins S5 and S12 are shown in cyan and gray, respectively. The backbone portion of the 900 tetraloop and that of helix 24 involved in intersubunit bridge B2c are represented as white sticks. solvent side of the $30 \mathrm{~S}$ subunit and interacts with residues at position 15, 18, and 19 of helix 1 . Protein S12 is located at the subunit interface and interacts with residues at position 909 in helix 27 and at position 913 at the junction of helix 27 and the central pseudoknot. Additional interactions involving helix 1 are revealed by the analysis of the crystal structure, but are not shown on Figure 4 for the sake of clarity. Helix 1 forms a coaxial stack with helix 28 , which connects the head of the $30 \mathrm{~S}$ subunit to its body. Nucleotides U12 and G15 contact, respectively, nucleotide C526 of helix 18 and nucleotide A1396 at the unction of helices 28 and 44 . Also, the crystal structure of the 30 S subunit complexed with streptomycin showed that the phosphate of U14 is involved in the binding of streptomycin Carter et al. 2000).

As illustrated in Figure 4, the 900 tetraloop and the central pseudoknot are positioned on the opposite ends of helix 27, indicating that the complementation between mutations in helix 1 and in the 900 tetraloop cannot result from a direct interaction. Site-directed cross-linking studies with 4-thiouridine identified a cross-link between residue U14 of helix 1 and the 903-904 region of helix 27, very close to the tetraloop (Juzumiene and Wollenzien 2001), but such a subunit. It is known that the top of helix 44 and neighing helices, such as helices 1 and 27, have some conformaof helix 2 and a drastic displacement of helices 1 and 27 . Rather, we propose that mutations in helix 1 affect the 900 tetraloop indirectly. Nucleotides $12-14$ are involved in base-triple interactions with a portion of helix 2 (see Fig. 1). It was previously shown that the U13A mutation causes a small increase in the binding of a probe complementary to the 915-921 region and in the reactivity of G917 to kethoxal (Pinard et al. 1995). The increased accessibility of residues within the central pseudoknot indicates that the U13A mutation destabilizes helix 2, making this pseudoknot more flexible. We hypothesize that the U12C and $\Delta \mathrm{U} 12$ mutations have a similar effect. The U12C and U13A mutations introduce potential Watson-Crick base pairs, whereas the $\Delta \mathrm{U} 12$ mutation, by removing one nucleotide from the apical loop of helix 1, may be expected to stabilize this loop. It is likely that the increased flexibility of the central pseudoknot results from an increased rigidity of helix 1 and its capping loop, favoring a transient disruption of helix 2 . The 
increased flexibility of the central pseudoknot caused by the U12C, $\Delta$ U12, and U13A mutations could decrease some of the constraints on the spatial orientation of helix 27, this effect being communicated via the connecting 913-915 segment. The increased mobility of helix 27 could facilitate the docking of the mutated 900 tetraloop into its receptor by avoiding the steric clash between G900 and G769 of helix 24 . Restoring the interaction between the 900 tetraloop and helix 24 would then favor the formation of the intersubunit bridge B2c, correcting the defect in subunit association caused by the A900G mutation. One could suggest that other mutations in the central pseudoknot could also increase its flexibility and compensate for the A900G mutation. However, no mutation was selected at positions other than 12-14, possibly because such mutations severely perturb the structure of the pseudoknot, thus strongly impairing the ribosome function. Proteins S5 and S12 also interact with the central pseudoknot, as described above. It is interesting to recall that the binding of either S5 or S12 to the 16S rRNA induces protection of bases A900 and A901 against dimethylsulfate attack (Stern et al. 1988). One likely interpretation of this protection, in light of the X-ray structure of the 30S subunit, is that these proteins, when interacting with 16S rRNA, help the reorientation of helix 27, so as to facilitate the docking of the tetraloop into its receptor. Proteins S5 and S12 could also contribute to the coupling of a movement of helix 27 with structural perturbations in the central pseudoknot. This appears to be especially true for protein S12, which interacts simultaneously with residue 909 in helix 27 and 913 in the central pseudoknot.

We previously proposed that the A900G mutation promotes the transition from the open to the closed conformation of the 30S subunit upon tRNA binding (Bélanger et al. 2004). If mutations in helix 1 facilitate the docking of the 900 tetraloop into its receptor, they also antagonize the effect of the A900G mutation on the transition from the open to the closed $30 \mathrm{~S}$ conformation. This would explain how the mutations in helix 1 correct the fidelity defect caused by the A900G mutation. In the absence of the A900G mutation, mutations in helix 1 do not impair the control of translational accuracy. This contrasts with poly(U)-directed in vitro assays that previously showed that the U13A mutation increases the control of translational fidelity (Pinard et al. 1994). It is likely that the slower kinetics of translation in vitro make the ribosomes more sensitive to subtle structural changes that do not have a significant effect in vivo. We also observed that the U13A and $\Delta \mathrm{U} 12$ mutations increase the levels of UGA readthrough, although they do not change the level of miscoding or frameshift. As mentioned above, a change in the readthrough level can reflect either a change in translational fidelity or a perturbation of the termination process (Murgola et al. 1995). The latter situation could account for the effect of the U13A and $\Delta \mathrm{U} 12$ mutations on the level of a stop codon readthrough.
Lastly, we observed that mutations in helix 1 alone mildly decrease the translational activity of the ribosome. This decrease in activity cannot be ascribed to a defect in the initiation of translation since, in that case, the proportion of mutant $30 \mathrm{~S}$ subunits in the $70 \mathrm{~S}$ and polysome populations would have been decreased. An attractive hypothesis is that these mutations could slightly perturb the translocation step. The central pseudoknot is indeed located at the junction of head, body, and platform of the $30 \mathrm{~S}$ subunit, close to the axis of rotation of the ratchet-like movement that occurs between the two ribosomal subunits during translocation (Frank and Agrawal 2000; Gao et al. 2003; Valle et al. 2003). The increased flexibility conferred to the central pseudoknot by mutations in helix 1 could alter the dynamics of the conformational movements that occur during translocation, resulting in the observed decrease in ribosome activity.

To conclude, our study reveals an indirect functional relationship between helix 1 and the 900-tetraloop capping helix 27 of $16 \mathrm{~S}$ rRNA. It will be interesting to investigate whether mutations in helix 1 can complement other mutations in helix 27 or in other regions of the $16 \mathrm{~S}$ rRNA. Unanticipated functional relationships can be readily detected with genetic complementation studies, as illustrated by the present work. The application of this approach to various strategic rRNA regions, combined with the information provided by highresolution X-ray structures, can efficiently contribute to further increase our understanding of ribosome function.

\section{MATERIALS AND METHODS}

\section{Reagents}

Restriction enzymes were from New England Biolabs, Fermentas, and Amersham Biosciences. Calf intestine alkaline phosphatase and T4 polynucleotide kinase were from Amersham Biosciences, T4 DNA ligase was from Fermentas, and M-MLV reverse transcriptase was from Invitrogen. Oligonucleotides were purchased from Integrated DNA Technology.

\section{Bacterial strains and plasmids}

All plasmids were maintained in E. coli DH5. Cultures were grown in LB medium with $100 \mu \mathrm{g} / \mathrm{mL}$ ampicillin (LB-Ap100). Plasmid pASS2-GFP2 was constructed in the laboratory of P.R. Cunningham and will be described elsewhere. Briefly, it contains a copy of the $16 \mathrm{~S}$ rRNA gene under control of the lacUV5 promoter, and the CAT and GFP genes, each under the control of a constitutive tryptophan promoter. The Shine-Dalgarno sequences of both CAT and GFP messengers are changed to $5^{\prime}$-AUCCC, and the anti-Shine-Dalgarno sequence of the $16 \mathrm{~S}$ rRNA is changed to $5^{\prime}$-GGGAU. The synthesis of plasmid-encoded 16S rRNA was induced by addition of IPTG to a final concentration of $1 \mathrm{mM}$. Plasmid pRNAluc2 contains a copy of the $r r n \mathrm{~B}$ operon under control of the inducible lacUV5 promoter and a reporter gene coding for the firefly luciferase (Bélanger et al. 2002). Plasmids pRNAluc2/UGA and pRNAluc $2 /-1$ contain a cassette in the beginning of the coding sequence of luciferase with a 
UGA stop codon or a -1 frameshift mutation. Plasmid pRNAluc2/ R218G contains a mutation that replaces arginine 218 of luciferase with a glycine (R218G), a mutation that inactivates luciferase (Viviani et al. 2002).

\section{Random mutagenesis of 165 rRNA and selection of compensatory mutations}

Plasmid pASS2-GFP2 bearing the A900G mutation was introduced into E. coli XL1-Red strain (Stratagene), following the manufacturer's instructions. Cells were plated on LB-Agar-Ap100 and grown for $24 \mathrm{~h}$ at $37^{\circ} \mathrm{C}$. About 200 colonies were picked into a single culture that was grown overnight at $37^{\circ} \mathrm{C}$, followed by a plasmid preparation. The pool of randomly mutated plasmids thus obtained was introduced into E. coli DH5. The culture was induced with $1 \mathrm{mM}$ IPTG for 3-1/2 h before plating on LB-Agar-Ap100 supplemented with $1 \mathrm{mM}$ IPTG and $175 \mu \mathrm{g} / \mathrm{mL}$ of chloramphenicol. After $24 \mathrm{~h}$ of incubation at $37^{\circ} \mathrm{C}$, surviving colonies were picked. To ensure that the complementary mutation is located in the 16S rRNA gene, BglII-BstEII or ApaI-ApaI fragments of the plasmids extracted from the surviving cells were cloned into pASS2GFP2/A900G. Clones that conserved the complementation phenotype were sequenced.

Amplification of the 16S rRNA gene from pASS2-GFP2/A900G was made with an error-prone PCR, using Taq DNA polymerase in the presence of $0.2 \mathrm{mM} \mathrm{MnCl}$. Primers were $16 \mathrm{~S}-5^{\prime} 5^{\prime}$-CCA AGTCTCAAGAGTGAACACG-3' $3^{\prime}$ and 16S-1542REV 5'-TAATCC CATGATCCAACCGC- $3^{\prime}$. The PCR fragments were cloned back into pASS2-GFP2/A900G, using the unique BstEII and BclI restriction sites located at each extremity of the 16S rRNA sequence. Ligation products were electroporated in E. coli DH5 cells and gain-of-function mutations were selected as described above.

The double U13A/A900G mutation was introduced into plasmid pASS2-GFP2 with a two-step PCR approach, using an overlap extension procedure (Ho et al. 1989). The final PCR product was cloned in pASS2-GFP2, using the two ApaI sites. PCR1 used primer U13A-REV 5'-AATTGAAGAGTATGATCATGGCTCAGATTGAA CG-3' (the U13A mutation is in bold) and primer A900G-FWD 5'-CCACCGCTTGTGCGGGCCCCCGTCAATTCATTTGAGTTTT AACCTCGCGGCCGTACTCC- $3^{\prime}$ (ApaI site is underlined and the A900G mutation is in bold). PCR2 used primer U13A-FWD $5^{\prime}$-GCGTTCAATCTGAGCCATGATC-3' and primer ApaI-REV 5'-GGCACAACAACTGGCGGGCAAACAGTCG-3' , located downstream from the second ApaI site. The final PCR was made using PCR1 and PCR2 as the template and primers A900G-FWD and ApaI-REV. A single mutant for which A900G is reverted to wild type was constructed with a standard PCR approach from each double mutant. The primers used were A900WT-FWD 5'-CCACC GCTTGTGCGGGCCCCCGTCAATTCATTTGAGTTTTAACCTT GCGGCCGTACTCC-3' in bold) and ApaI-REV described above. The PCR product was cloned in plasmid pASS2-GFP2, using the two ApaI sites.

\section{GFP and luciferase assays}

Overnight cultures from plasmid-containing cells grown in LB-Ap100 were diluted to an optical density of 0.1 at $600 \mathrm{~nm}$ in $2 \mathrm{~mL}$ and incubated for $1 \mathrm{~h}$ at $37^{\circ} \mathrm{C}$. The cultures were then induced by adding $1 \mathrm{mM}$ IPTG and incubated for another $5 \mathrm{~h}$ at $37^{\circ} \mathrm{C}$ for
GFP assays and $3-1 / 2 \mathrm{~h}$ at $37^{\circ} \mathrm{C}$ for luciferase assays. GFP and luciferase assays were carried out as described (Bélanger et al. 2002).

\section{Analysis of mutant 16S rRNA distribution}

Cells containing pASS2-GFP2 and its mutant derivatives were induced for 3-1/2 h, and ribosomal particles were isolated from bacterial lysates obtained by freezing and thawing, following a standard procedure. Lysates were applied onto a 15\%-40\% (w/v) linear sucrose gradient in $20 \mathrm{mM}$ Tris- $\mathrm{HCl}$ (pH 7.8), $5 \mathrm{mM} \mathrm{MgCl}_{2}, 100 \mathrm{mM}$ $\mathrm{NH}_{4} \mathrm{Cl}, 2 \mathrm{mM}$ DTT, and centrifuged at 20,000 rpm for $20 \mathrm{~h}$ in a Beckman SW 28 rotor at $4^{\circ} \mathrm{C}$. Fractions containing free $30 \mathrm{~S}$ subunits, $70 \mathrm{~S}$ ribosomes, and polysomes were detected at $260 \mathrm{~nm}$ and collected with an ISCO gradient fractionator. The proportion of plasmidencoded 16S rRNA in RNA isolated from these fractions and from the crude lysate was determined by primer extension, as described (Sigmund et al. 1988; Bélanger et al. 2002).

\section{ACKNOWLEDGMENTS}

We thank Pascale Legault, Mélissa Léger, Stephen Michnick, and Sergey Steinberg for critical reading of this manuscript and for stimulating comments. This work was supported by a grant from the Canadian Institutes of Health Research (CIHR) to L.B-G. and National Institutes of Health (NIH) grant GM52896 to P.R.C. F.B. held a scholarship from the Fonds pour la Formation des Chercheurs et l'Aide à la Recherche (FCAR) and a travel grant from the Gouvernement du Québec.

Received January 26, 2005; accepted March 4, 2005.

\section{REFERENCES}

Ban, N., Nissen, P., Hansen, J., Moore, P.B., and Steitz, T.A. 2000. The complete atomic structure of the large ribosomal subunit at $2.4 \AA$ resolution. Science 289: 905-920.

Bélanger, F., Léger, M., Saraiya, A.A., Cunningham, P.R., and BrakierGingras, L. 2002. Functional studies of the 900 tetraloop capping helix 27 of 16S ribosomal RNA. J. Mol. Biol. 320: 979-989.

Bélanger, F., Gagnon, M.G., Steinberg, S.V., Cunningham, P.R., and Brakier-Gingras, L. 2004. Study of the functional interaction of the 900 tetraloop of $16 \mathrm{~S}$ ribosomal RNA with helix 24 within the bacterial ribosome. J. Mol. Biol. 338: 683-693.

Brink, M.F., Verbeet, M.P., and de Boer, H.A. 1993. Formation of the central pseudoknot in 16S rRNA is essential for initiation of translation. EMBO J. 12: 3987-3996.

Carter, A.P., Clemons, W.M., Brodersen, D.E., Morgan-Warren, R.J., Wimberly, B.T., and Ramakrishnan, V. 2000. Functional insights from the structure of the $30 \mathrm{~S}$ ribosomal subunit and its interactions with antibiotics. Nature 407: 340-348.

Druzina, Z. and Cooperman, B.S. 2004. Photolabile anticodon stemloop analogs of tRNA ${ }^{\text {Phe }}$ as probes of ribosomal structure and structural fluctuation at the decoding center. RNA 10: 1550-1562.

Frank, J. and Agrawal, R.K. 2000. A ratchet-like inter-subunit reorganization of the ribosome during translocation. Nature 406: 318-322.

Gabashvili, I.S., Whirl-Carrillo, M., Bada, M., Banatao, D.R., and Altman, R.B. 2003. Ribosomal dynamics inferred from variations in experimental measurements. RNA 9: 1301-1307.

Gao, H., Sengupta, J., Valle, M., Korostelev, A., Eswar, N., Stagg, S.M., Van Roey, P., Agrawal, R.K., Harvey, S.C., Sali, A., et al. 2003. Study of the structural dynamics of the E. coli 70 S ribosome using real-space refinement. Cell 113: 789-801. 
Gutell, R.R., Larsen, N., and Woese, C.R. 1994. Lessons from an evolving rRNA: $16 \mathrm{~S}$ and $23 \mathrm{~S}$ rRNA structures from a comparative perspective. Microbiol. Rev. 58: 10-26.

Harms, J., Schluenzen, F., Zarivach, R., Bashan, A., Gat, S., Agmon, I., Bartels, H., Franceschi, F., and Yonath, A. 2001. High resolution structure of the large ribosomal subunit from a mesophilic eubacterium. Cell 107: 679-688.

Ho, S.N., Hunt, H.D., Horton, R.M., Pullen, J.K., and Pease, L.R. 1989. Site-directed mutagenesis by overlap extension using the polymerase chain reaction. Gene 77: 51-59.

Juzumiene, D.I. and Wollenzien, P. 2001. Arrangement of the central pseudoknot region of $16 \mathrm{~S}$ rRNA in the $30 \mathrm{~S}$ ribosomal subunit determined by site-directed 4-thiouridine crosslinking. RNA 7: 71-84.

Kössel, H., Hoch, B., and Zeltz, P. 1990. Alternative base pairing between $5^{\prime}$ - and $3^{\prime}$-terminal sequences of small subunit RNA may provide the basis of a conformational switch of the small ribosomal subunit. Nucleic Acids Res. 18: 4083-4088.

Leclerc, D. and Brakier-Gingras, L. 1991. A conformational switch involving the 915 region of Escherichia coli 16 S ribosomal RNA. FEBS Lett. 279: 171-174.

Lee, K., Varma, S., SantaLucia Jr., J., and Cunningham, P.R. 1997. In vivo determination of RNA structure-function relationships: Analysis of the 790 loop in ribosomal RNA. J. Mol. Biol. 269: 732-743.

Lodmell, J.S. and Dahlberg, A.E. 1997. A conformational switch in Escherichia coli 16S ribosomal RNA during decoding of messenger RNA. Science 277: 1262-1267.

Morosyuk, S.V., Lee, K., SantaLucia Jr., J., and Cunningham, P.R. 2000. Structure and function of the conserved 690 hairpin in Escherichia coli $16 \mathrm{~S}$ ribosomal RNA: Analysis of the stem nucleotides. J. Mol. Biol. 300: 113-126.

Morosyuk, S.V., SantaLucia, Jr., J., and Cunningham, P.R. 2001. Structure and function of the conserved 690 hairpin in Escherichia coli $16 \mathrm{~S}$ ribosomal RNA. III. Functional analysis of the 690 loop. J. Mol. Biol. 307: 213-228.

Murgola, E.J., Pagel, F.T., Hijazi, K.A., Arkov, A.L., Xu, W., and Zhao, S.Q. 1995. Variety of nonsense suppressor phenotypes associated with mutational changes at conserved sites in Escherichia coli ribosomal RNA. Biochem. Cell Biol. 73: 925-931.

Ogle, J.M., Brodersen, D.E., Clemons Jr., W.M., Tarry, M.J., Carter, A.P., and Ramakrishnan, V. 2001. Recognition of cognate transfer RNA by the 30S ribosomal subunit. Science 292: 897-902.

Ogle, J.M., Murphy, F.V., Tarry, M.J., and Ramakrishnan, V. 2002. Selection of tRNA by the ribosome requires a transition from an open to a closed form. Cell 111: 721-732.

Ogle, J.M., Carter, A.P., and Ramakrishnan, V. 2003. Insights into the decoding mechanism from recent ribosome structures. Trends Biochem. Sci. 28: 259-266.

Pinard, R., Payant, C., Melancon, P., and Brakier-Gingras, L. 1993. The $5^{\prime}$ proximal helix of $16 \mathrm{~S}$ rRNA is involved in the binding of streptomycin to the ribosome. FASEB J. 7: 173-176.

Pinard, R., Cote, M., Payant, C., and Brakier-Gingras, L. 1994. Positions 13 and 914 in Escherichia coli 16S ribosomal RNA are involved in the control of translational accuracy. Nucleic Acids Res. 22: 619-624.
Pinard, R., Payant, C., and Brakier-Gingras, L. 1995. Mutations at positions 13 and/or 914 in Escherichia coli 16S ribosomal RNA interfere with the initiation of protein synthesis. Biochemistry 34: 9611-9616.

Poot, R.A., Pleij, C.W., and van Duin, J. 1996. The central pseudoknot in $16 \mathrm{~S}$ ribosomal RNA is needed for ribosome stability but is not essential for $30 \mathrm{~S}$ initiation complex formation. Nucleic Acids Res. 24: $3670-3676$

Poot, R.A., van den Worm, S.H., Pleij, C.W., and van Duin, J. 1998. Base complementarity in helix 2 of the central pseudoknot in $16 \mathrm{~S}$ rRNA is essential for ribosome functioning. Nucleic Acids Res. 26: 549-553.

Rodriguez-Correa, D. and Dahlberg, A.E. 2004. Genetic evidence against the $16 \mathrm{~S}$ ribosomal RNA helix 27 conformational switch model. RNA 10: 28-33.

Schluenzen, F., Tocilj, A., Zarivach, R., Harms, J., Gluehmann, M., Janell, D., Bashan, A., Bartels, H., Agmon, I., Franceschi, F. et al. 2000. Structure of functionally activated small ribosomal subunit at $3.3 \AA$ resolution. Cell 102: 615-623.

Sigmund, C.D., Ettayebi, M., Borden, A., and Morgan, E.A. 1988. Antibiotic resistance mutations in ribosomal RNA genes of Escherichia coli. Methods Enzymol. 164: 673-690.

Stern, S., Powers, T., Changchien, L.M., and Noller, H.F. 1988. Interaction of ribosomal proteins S5, S6, S11, S12, S18 and S21 with 16 S rRNA. J. Mol. Biol. 201: 683-695.

Valle, M., Zavialov, A., Sengupta, J., Rawat, U., Ehrenberg, M., and Frank, J. 2003. Locking and unlocking of ribosomal motions. Cell 114: $123-134$

van Heel, M. 2000. Unveiling ribosomal structures: The final phases. Curr. Opin. Struct. Biol. 10: 259-264.

VanLoock, M.S., Agrawal, R.K., Gabashvili, I.S., Qi, L., Frank, J., and Harvey, S.C. 2000. Movement of the decoding region of the $16 \mathrm{~S}$ ribosomal RNA accompanies tRNA translocation. J. Mol. Biol. 304: 507-515.

Velichutina, I.V., Dresios, J., Hong, J.Y., Li, C., Mankin, A., Synetos, D., and Liebman, S.W. 2000. Mutations in helix 27 of the yeast Saccharomyces cerevisiae $18 \mathrm{~S}$ rRNA affect the function of the decoding center of the ribosome. RNA 6: 1174-1184.

Vila-Sanjurjo, A., Ridgeway, W.K., Seymaner, V., Zhang, W., Santoso, S. Yu, K., and Cate, J.H. 2003. X-ray crystal structures of the WT and a hyper-accurate ribosome from Escherichia coli. Proc. Natl. Acad. Sci. 100: $8682-8687$.

Viviani, V.R., Uchida, A., Viviani, W., and Ohmiya, Y. 2002. The influence of Ala243 (Gly247), Arg215 and Thr226 (Asn230) on the bioluminescence spectra and $\mathrm{pH}$-sensitivity of railroad worm, click beetle and firefly luciferases. Photochem. Photobiol. 76: 538-544.

Wimberly, B.T., Brodersen, D.E., Clemons Jr., W.M., MorganWarren, R.J., Carter, A.P., Vonrhein, C., Hartsch, T., and Ramakrishnan, V. 2000. Structure of the 30 S ribosomal subunit. Nature 407: 327-339.

Yusupov, M.M., Yusupova, G.Z., Baucom, A., Lieberman, K., Earnest, T.N., Cate, J.H., and Noller, H.F. 2001. Crystal structure of the ribosome at $5.5 \AA$ resolution. Science 292: $883-896$. 

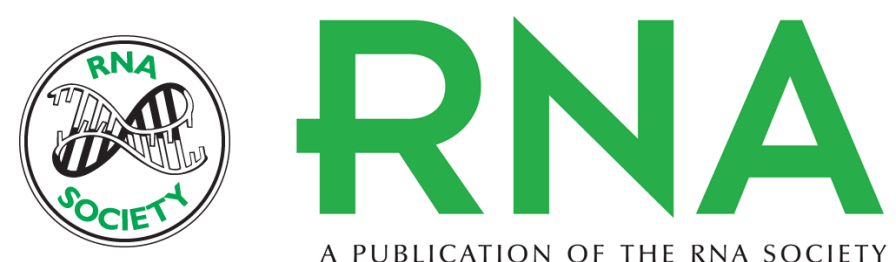

A PUBLICATION OF THE RNA SOCIETY

\section{A functional relationship between helix 1 and the 900 tetraloop of $16 \mathrm{~S}$ ribosomal RNA within the bacterial ribosome}

FRANÇOIS BÉLANGER, GABRIEL THÉBERGE-JULIEN, PHILIP R. CUNNINGHAM, et al.

RNA 2005 11: 906-913

References This article cites 40 articles, 11 of which can be accessed free at:

http://rnajournal.cshlp.org/content/11/6/906.full.html\#ref-list-1

\section{License}

Email Alerting Service

Receive free email alerts when new articles cite this article - sign up in the box at the top right corner of the article or click here. 